\title{
History Matching of a Stochastic Model of Field-Scale Fractures: Methodology and Case Study
}

\author{
S. Jenni' ${ }^{1,2}$, L.Y. Hu' ${ }^{1}$, R. Basquet ${ }^{1,3}$, G. de Marsily ${ }^{4}$ and B. Bourbiaux ${ }^{1}$ \\ 1 Institut Français du Pétrole, IFP, 1-4 avenue de Bois-Préau, 92852 Rueil-Malmaison Cedex - France \\ 2 Schlumberger Water Services, Le Palatin, 92936 Paris-La Défense Cedex - France \\ 3 Statoil ASA, Forusbeen 35, 4035 Stavanger - Norway \\ 4 Université Paris VI, 4 Place Jussieu, 75252 Paris Cedex 05 - France \\ email: sjenni@la-defense.oilfield.slb.com - l-ying.hu@ifp.fr
}

\begin{abstract}
Résumé - Calage d'historique d'un modèle stochastique de fractures à l'échelle réservoir : méthodologie et cas d'étude - Cet article vise le calage d'historique d'un modèle stochastique de fractures à grande échelle mais au dessous de la résolution sismique, à savoir les failles sub-sismiques et les couloirs de fracturation. D'abord, nous proposons un modèle stochastique de type objet pour décrire les aspects géologiques des fractures à grande échelle. Ce modèle prend en compte des contraintes statiques dérivées des attributs sismiques, des considérations géomécaniques, de l'information structurale (courbure), etc. Ensuite, nous passons en revue une procédure de mise à échelle pour construire un modèle de simulation d'écoulement de fluides en présence des réseaux de fractures à grande échelle. Enfin, nous présentons un algorithme pour graduellement déplacer et déformer des fractures stochastiques dans le champ réservoir tout en préservant leur cohérence avec les contraintes statiques (position des failles sismiques, cartes de densité et d'orientation de fractures), par lequel différentes réalisations du réseau stochastique de fractures peuvent être obtenues. Tous ces éléments sont intégrés dans une procédure inverse pour caler le modèle stochastique des réseaux de fractures à grande échelle à des données d'écoulement diphasique.

La méthodologie ci-dessus est appliquée à un réservoir fracturé. Nous construisons un réseau de fracture à l'échelle du champ contraint par la carte de densité de fractures et les orientations des deux familles de fractures. Puis, nous effectuons un calage aux données de water-cut dans quatre zones du champ. Différentes procédures de calage sont examinées : optimisation globale qui permet une amélioration générale du calage du modèle aux données de production, et optimisation locale qui améliore davantage le calage de chaque puits. Les résultats démontrent la validité de la méthodologie proposée.
\end{abstract}

Abstract - History matching of a stochastic model of field-scale fractures: methodology and case study - This paper focuses on the history matching of stochastic models of large-scale fractures under seismic resolution, namely sub-seismic faults and fracture swarms. First, we propose an object-based stochastic model for describing geological features of large-scale fractures. This model accounts for static constraints derived from seismic attributes, fault-related-strain-field, structural information (curvature), etc. Second, we review an upscaling procedure for performing fluid flow simulation in the presence of networks of large-scale fractures. Third, we present an algorithm for gradually moving and deforming 
stochastic fractures in the reservoir field while preserving their consistency with static constraints (location of seismic fractures, fracture density and orientation maps), whereby different realizations of the stochastic fracture network can be obtained. All these elements are integrated in an inverse procedure for calibrating stochastic models of large-scale fracture networks to hydrodynamic two-phase flow data.

The above methodology is applied to an actual fractured reservoir. We build a field-scale fracture network constrained to the fracture density map and the orientations of the two fracture sets. Then we perform history matching of water-cut data in four zones of the field. Different calibration procedures are tested: global optimization that allows a general improvement of the model calibration to production data, and local calibration that further improves the match of each well. The results show the validity of the proposed methodology.

\section{INTRODUCTION}

Fractures radically affect sub-surface fluid flow and can act as preferential flow paths if they remain open after their formation or as flow barriers if they are sealed by impervious material. The unexpected production behavior of many fields (early water breakthrough, compartmentalization, dual permeability effects, etc.), arising from an insufficient consideration of fracture effects on flow, emphasizes the need for better characterizing the distribution of fractures at various scales and transferring the meaningful part of this information into field flow simulation models.

During the last decade, tremendous advances have been made in the modeling of fractured reservoirs, both with respect to geological characterization and fluid flow simulation. Modern geological modeling of fracture networks [1, 2], based on a fractal or stochastic approach, is able to integrate local data from well-bore imaging and field scale data derived from seismic attributes, fault-related-strain-field, structural information (curvature), etc. These geological (static) models of fracture networks can now be turned into flow models for well-test simulation or full-field simulation, through practical upscaling solutions [3, 4]. Constraining these models to hydrodynamic data from well-tests and production history is the natural next step in the development of an effective modeling methodology for fractured reservoirs.

Natural fracturing is a typical multi-scale phenomenon. Considering the impact of fractures on fluid flow, reservoir engineers generally classify fractures of different scales into two major classes: large-scale fractures cross-cutting the reservoir and small-scale fractures preferentially located in given reservoir layers. Small-scale fractures can be homogenized into a geo-cellular model while large-scale fractures must be directly taken into account in flow simulations.

This paper focuses on large-scale fractures. On the basis of existing methods for geological modeling [2], fluid flow simulation [5] and deformation of object-based stochastic model [6], we propose an integrated methodology for history matching of stochastic models of large-scale fracture networks to production data. This methodology is applied to a North Africa Field and the results are presented.

\section{GEOLOGICAL MODELING OF LARGE-SCALE FRACTURES}

The methodology for geological modeling of fracture networks is extensively described by Cacas et al. [2] and Bourbiaux et al. [7]. Large-scale fractures include faults above seismic resolution, sub-seismic faults or clustered joints of large vertical extent, called fracture swarms. These fractures are split into different sets according to specified geometrical properties such as orientation or spacing, in relation with past tectonic episodes. All fracture sets are described separately and then merged into a global model that incorporates:

- a deterministic description of large-scale faults above seismic resolution,

- a stochastic description of other faults and fractures under seismic resolution, namely sub-seismic faults or fracture swarms.

We consider stochastic vertical fractures that cross the entire reservoir. The information for constraining each stochastic fracture set consists of:

- an orientation map based on seismic fault information and well data. This map allows to model spatial variation of fracture orientation;

- statistical parameters related to the distribution function of fracture lengths, which is inferred from fault analysis;

- fracture density map (or probability map of fracture occurrence) derived from geological, geomecanical and seismic information known over the entire field (seismic attributes, fault-related strain field, structural information such as curvature intensity map etc). The methodology for building such a map can be found, for instance, in Gauthier et al. [8]. This map controls both the location and the extension of stochastic fractures.

Fractures of a given fracture set are generated sequentially in three steps. For each fracture,

- first we randomly generate its location (fracture seed) according to a Poisson point pattern (process) with a regionalized intensity [9], which is proportional to the fracture density map;

- second, its length is drawn from the fracture length distribution; 


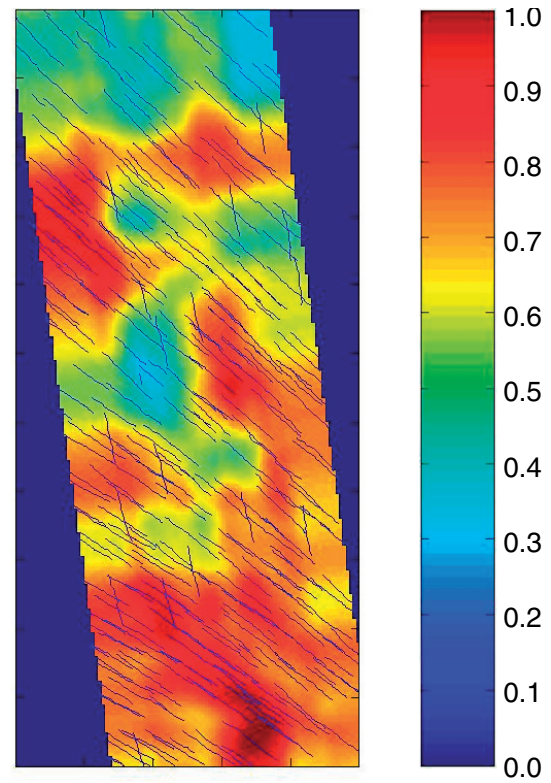

Figure 1

Realization of a stochastic model of fracture network with the corresponding fracture density map ( 1 colour for highest density and 0 for lowest density).

- third, we propagate the fracture, from the seed, under geological constraints. A fracture growth process conditioned to the fracture orientation map governs the incremental propagation of a broken line away from the fracture seed. The growth process is continued until the fracture length is reached. The fracture propagation is also constrained to the fracture density map. Once a fracture is generated, the density map is updated in the neighborhood of the fracture to account for fracture interactions (repulsive behavior of large faults, clustering, etc.).

Following the above algorithm, we can build realistic stochastic fracture networks constrained to geological (static) data. Figure 1 shows an example of such network. There are two independent fracture sets, one oriented around N120-130 and the other around N170. They are constrained by the fracture density map shown in the same figure. The next step is to calibrate such stochastic fracture network to production history.

\section{FLUID FLOW SIMULATION}

History matching applications need an accurate representation of fracture networks in fluid flow simulation models. For instance, a small difference of fracture locations can result in connecting or disconnecting flow paths between the wells and thus highly affecting the hydrodynamic behavior of the reservoir model. In the present methodology for history matching, we use a conventional dual porosity simulator with an upscaling procedure that does not mixture large-scale fractures with the matrix medium and the small-scale fractures (smaller than the cell size of the fluid flow simulation grid). This approach for fluid flow simulation was successfully applied to a giant Middle East oil field with large-scale fractures [5].
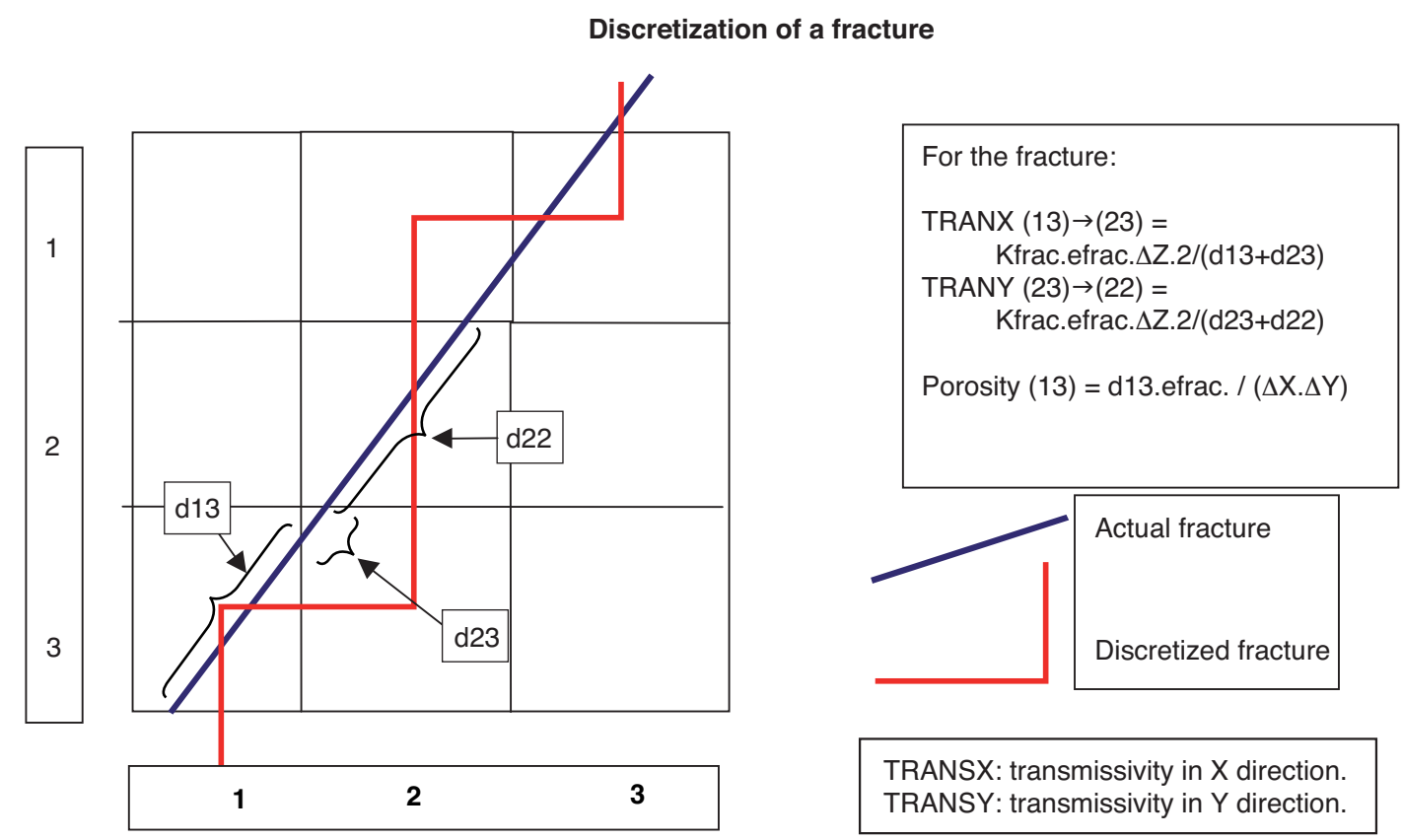

Figure 2

Procedure for determining the effective fracture porosity and transmissivity for the (fracture) simulation grid [5]. 


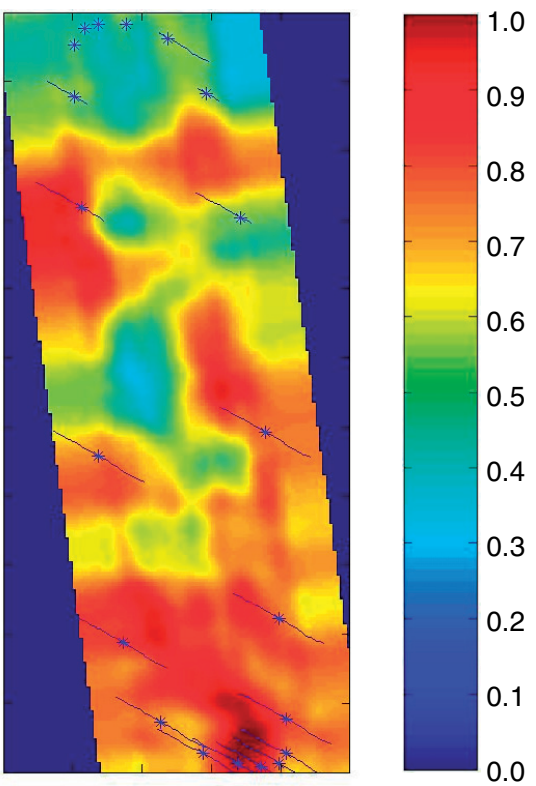

Figure 3

Gradual deformation of a fracture constrained to a fracture density map: successive locations and lengths of a given fracture during the deformation by changing the parameter $t$ of a realization chain.

Upscaling aims at defining reservoir properties at the simulation grid scale. A sound upscaling procedure should reproduce, at the coarser scale of the simulation grid, the same fluid flow behavior as if the discrete fracture network were used. In a dual-medium context, the problem is decoupled, since there are actually two simulation grids: the matrix grid and the fracture grid. In this paper, we assume that the upscaling procedures are known for the matrix medium and the small-scale fractures, we focus on the upscaling method for large-scale fractures (seismic and sub-seismic faults), as explained bellow.

The method is based on the direct calculation of the transmissivity terms in the fracture grid. First the discrete fracture network is superimposed onto the simulation grid. The effective length and the location of each fracture segment in each cell are computed. Each fracture is approximated by a discretized fracture as illustrated in Figure 2. Then the effective fracture porosity $\phi_{i, j}$ is computed as:

$$
\phi_{i, j}=\left(d_{i, j} \times e_{\text {frac }}\right) /\left(\Delta X_{i, j} \times \Delta Y_{i, j}\right)
$$

where:

$d_{i, j}:$ effective length of the fracture in cell $(i, j)$,

$e_{\text {fra }}$ : fracture thickness,

$\Delta X_{i, j}$ : cell dimension in $X$ direction,

$\Delta Y_{i, j}$ : cell dimension in $Y$ direction.

The procedure allows also for the direct calculation of the transmissivity terms in the fracture grid. When a fracture crosses two adjacent cells $(i, j)$ and $(k, l)$, the transmissivity is given by:

$$
T_{(i, j) \rightarrow(k, l)}=\left(K_{\text {frac }} \times e_{\text {frac }} \times \Delta Z_{i, j} \times 2\right) /\left(d_{i, j}+d_{k, l}\right)
$$

where:

$K_{\text {frac }}:$ fracture intrinsic permeability,

$\Delta Z_{i, j}$ : cell dimension in $Z$ direction.

If there are several fractures in a given cell, the relevant properties are added.

Another important parameter in a dual media simulation is the matrix block size, which controls the fluid exchanges between matrix and fractures. A practical method for computing the matrix block size is described in Bourbiaux et al. [3].

\section{HISTORY MATCHING OF STOCHASTIC FRACTURES}

History matching of stochastic fracture networks can be formulated as an optimization problem. We define an objective function that measures the difference between the production data observed in the actual field and the corresponding responses of a stochastic model obtained through flow simulation. Then we minimize this objective function by modifying an initial realization of the stochastic model. An effective optimization procedure requires the development of algorithms for modifying the model realization while maintaining its consistency with the geological (static) constraints. The algorithm for modifying fracture networks is based on the gradual deformation of object-based Boolean models [6]. We recall here the basic principle of this algorithm.

\subsection{Migration of General Poisson Point Patterns}

Fracture locations (seeds) follow a Poisson point pattern. Consider first a stationary Poisson point pattern. Starting from two independent points $x_{1}$ and $x_{2}$ uniformly distributed in the $m$ dimensional field $[0,1]^{m}$, we define a migration trajectory between $x_{1}$ and $x_{2}$ according to the gradual deformation algorithm:

$$
x(t)=G\left[G^{-1}\left(x_{1}\right) \cos t+G^{-1}\left(x_{2}\right) \sin t\right]
$$

where $G$ stands for the standard Gaussian cumulative distribution function. It can be shown that for any $t, x(t)$ is a uniform point in $[0,1]^{m}$. When two points are fixed, the migration trajectory between them is entirely determined.

However, for a non-stationary Poisson point pattern, the points are not uniformly distributed in space. But by use of the inverse distribution method to simulate a non-stationary Poisson point pattern, the location of each point still corresponds to a uniform vector. Consequently we can apply the gradual deformation method to migrate objects of a non-stationary Boolean model [6]. 


\subsection{Gradual Deformation of Stochastic Fractures}

Unlike the classical Boolean model, the stochastic model of large-scale fracture networks presented in this paper uses directly the fracture density map as the intensity function of the Poisson point pattern, and the fracture extension is constrained to the same density map. Therefore, fracture location and extension are dependent.

To modify the fracture network, we simply migrate fracture seeds using the gradual deformation algorithm defined by Equation (3). Then, starting from the modified fracture seeds, we apply the geological modeling procedure to re-generate the fractures. The migration of the locations of fractures results in subsequent modification of their extensions.

This method allows for gradual deformation of a stochastic fracture network in the reservoir field while preserving their consistency with geological constraints (location of seismic fractures, fracture density and orientation maps). Figure 3 shows an example of the migration of a fracture. We note that in this example, the gradual migration of the fracture results in a gradual variation of its length to honor the fracture density map.

The simultaneous migration of all fractures of a realization according to Equation (3) defines a chain of realizations of

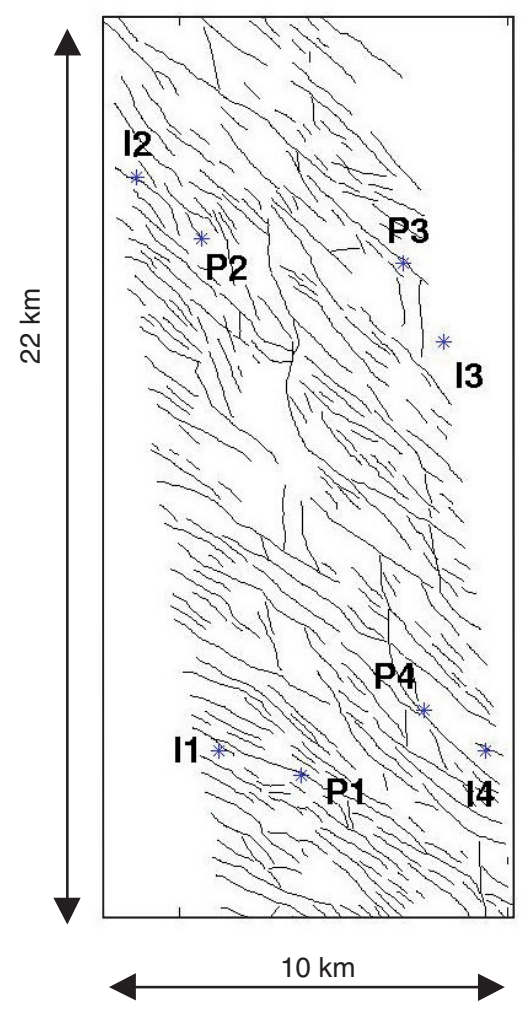

Figure 4

Reference fracture network and well locations (P: Producer; I: Injector). the fracture network. Then we optimize the objective function with respect to the deformation parameter $t$ to search for the realization in this chain that best fits the production data of the reservoir. If the optimized realization does not reduce the objective function to a low enough level, we can repeat the procedure by combining the optimized realization with another independent realization. The procedure is repeated until obtaining a satisfactory calibration.

Because of the discrete nature of object-based models, the objective function of a realization chain is in general not differentiable with respect to the gradual deformation parameter t. Consequently, gradient based optimization algorithms cannot be applied and we must turn to algorithms for optimizing non-differentiable functions such us the simplex method [10].

Different fracture sets of the geological model presented in this paper are generated independently. For each fracture set, a gradual deformation parameter $t_{i}$ controls the migration of the fractures. Then, the number of fitting parameters equals the number of fracture sets.

Finally, from different initial realizations of the stochastic fracture network, one can obtain different realizations calibrated to the production history. These calibrated realizations serve for uncertainty evaluation in future production forecasts.

\section{CASE STUDY: CALIBRATION OF A FRACTURE NETWORK TO WATER-CUT DATA}

The field case example is intended to illustrate the above methodology for calibrating stochastic fracture networks to production data. The fracture network is derived from a real case but the production history (water-cut) used for the inversion is simulated.

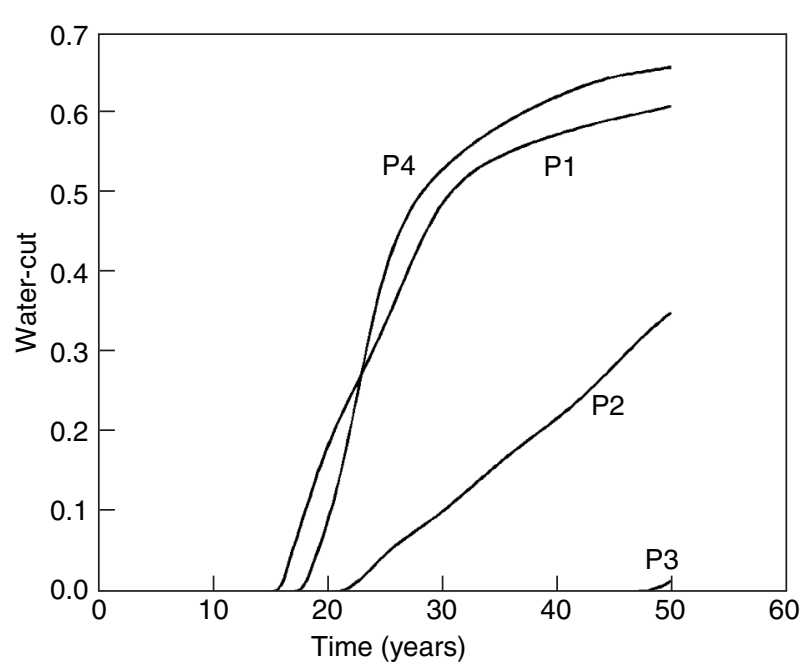

Figure 5

Water-cut versus time (year) obtained from fluid flow simulation for the reference fracture network. 


\subsection{Reference Case}

The case study concerns an oil-bearing field of $10 \mathrm{~km} \times 22 \mathrm{~km}$ large. In this field, a carbonate sequence of late Paleocene age is subdivided into an oil-producing upper reservoir unit and a mostly water-bearing lower interval. At the top of the reservoir, the faults are mainly oriented N120130. This fault network developed just above the deep main trends, which strike preferentially N170. Subsequently, the field structure can be explained by the reactivation of deep basement faults within an oblique extension regime [8]. This structural style results in the dominant oblique northwest/southeast normal faults and in N170 secondary faults and flexures at the top of the reservoir (Figure 4).

We consider a production scheme with four water injectors and four producers located in four different zones of the field (Figure 4). We use the above upscaling procedure for building a fluid flow simulation model with 100 and 220 cells along the $X$ and $Y$ axes, respectively. Due to the dominance of the faults oriented N120-130, the matrix-fracture exchange is considered as one-dimensional. Thus, if $N$ fractures are located in a cell the matrix block size in one of the three directions is $D /(N+1)$, where $D$ stands for the cell dimension in this direction. In the other two directions, the matrix block size is infinite.

The flow simulation using a dual porosity and dual permeability simulator is conducted over 50 years and the water-cut curves of the four producers are shown on Figure 5. The petrophysical properties used are the following:

$$
\begin{aligned}
K_{\text {mat }} & =50 \mathrm{mD} \\
K_{\text {frac }} & =3.5 \times 10^{6} \mathrm{mD} \\
e_{\text {frac }} & =0.1 \mathrm{~m} \\
\phi_{\text {mat }} & =15 \% \\
H & =10 \mathrm{~m}
\end{aligned}
$$

a)

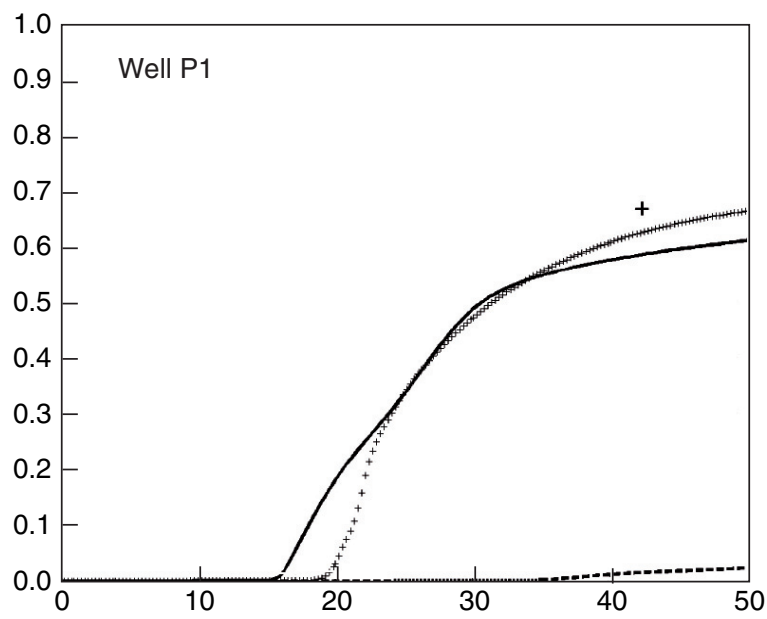

c)

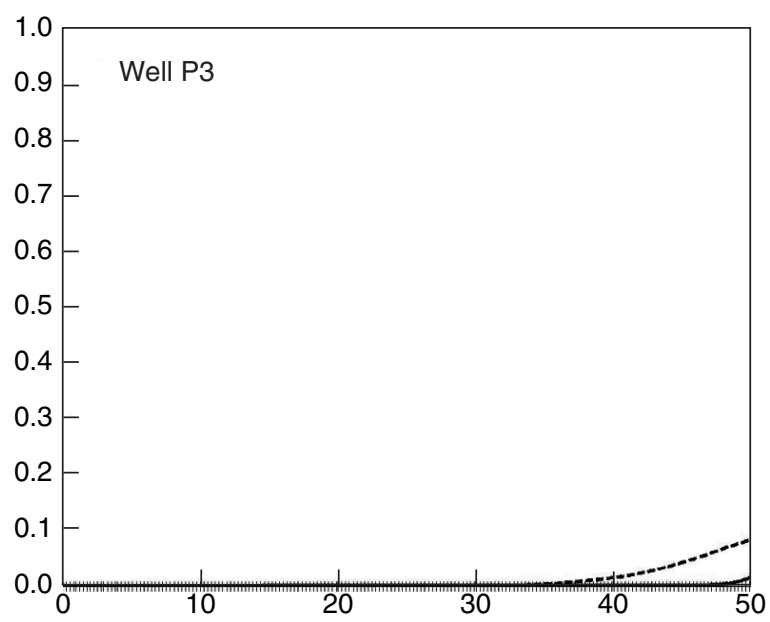

b)

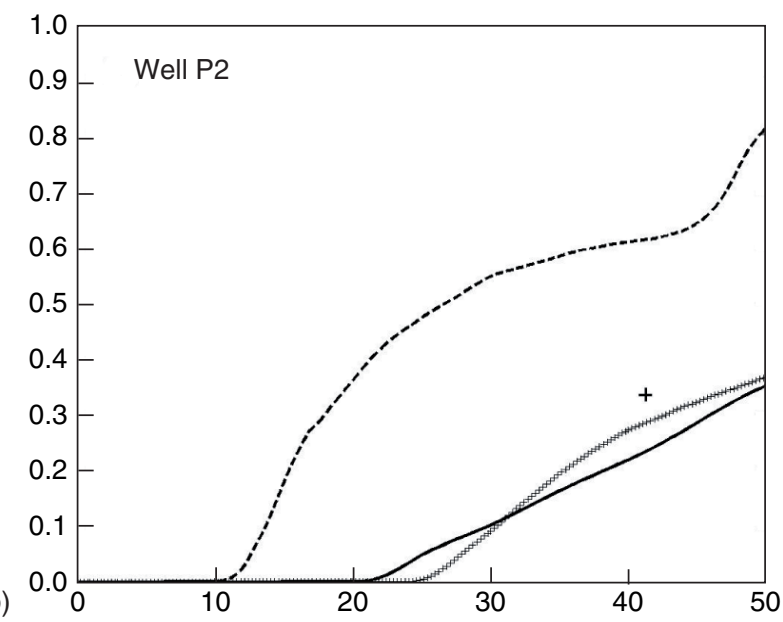

d)

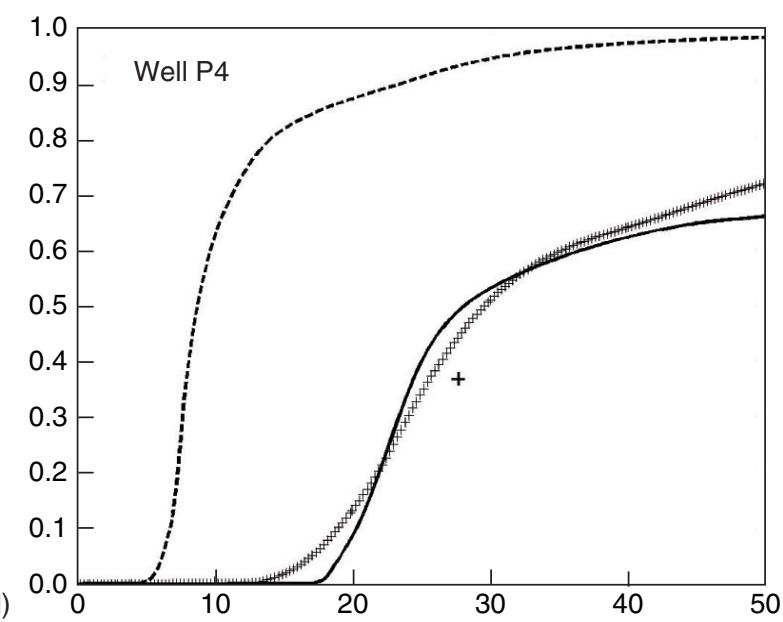

Figure 6

Calibration of the first realization: comparison of the water-cut curves of the reference fracture network $(-)$, the initial realization $(--)$ and the optimal realization $(+++)$. 


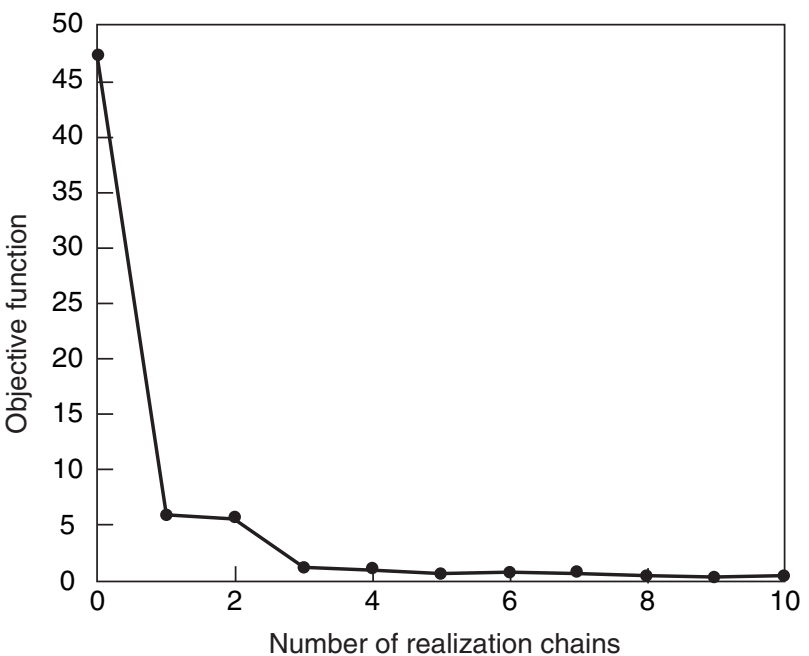

Figure 7

First example: evolution of the objective function when optimizing realization chains.

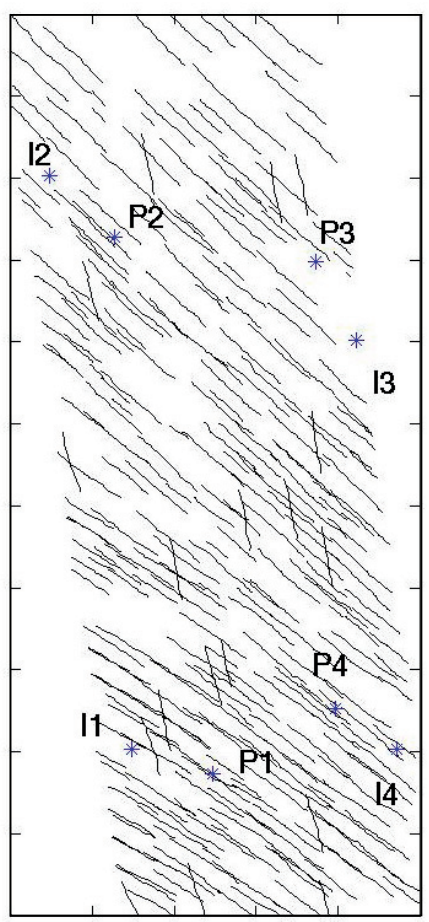

Figure 8

Calibration of the first realization: realization of the stochastic fracture network after global optimization.

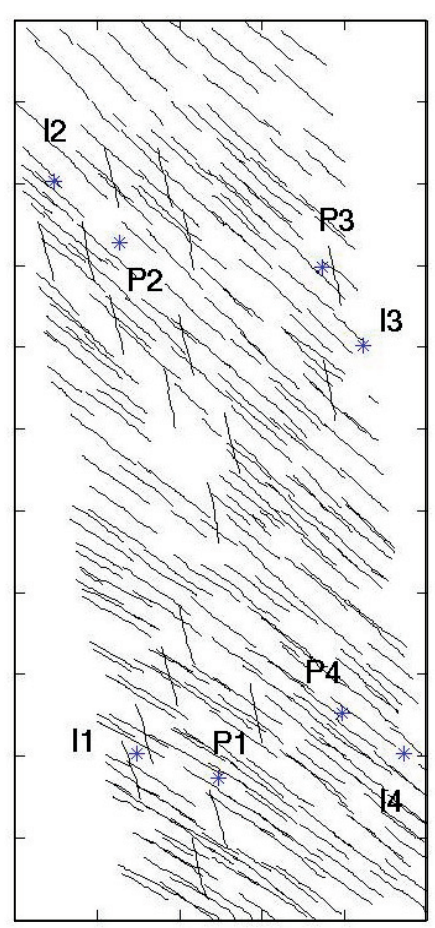

Figure 9

Calibration of the second realization: initial realization of the stochastic fracture network.

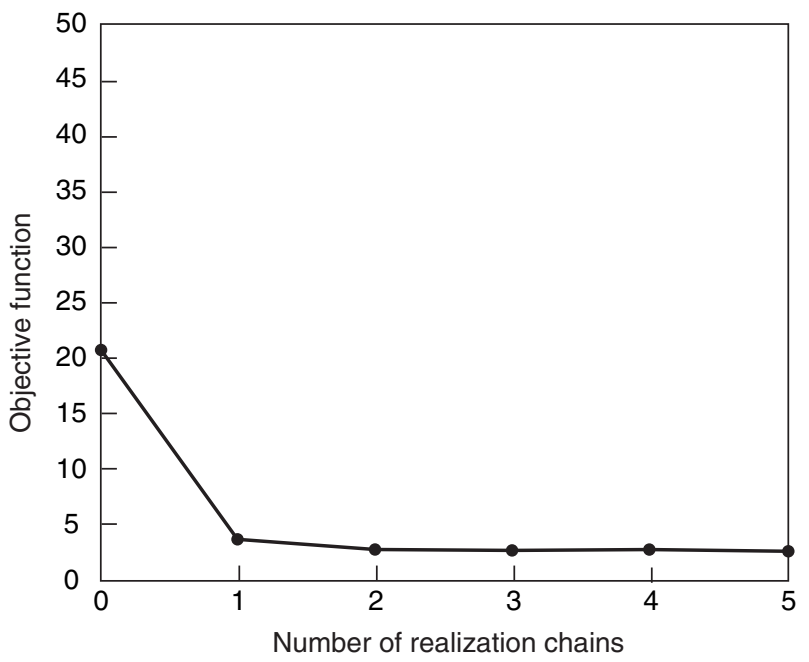

Figure 10

Calibration of the second realization: evolution of the objective function when optimizing realization chains. 
a)
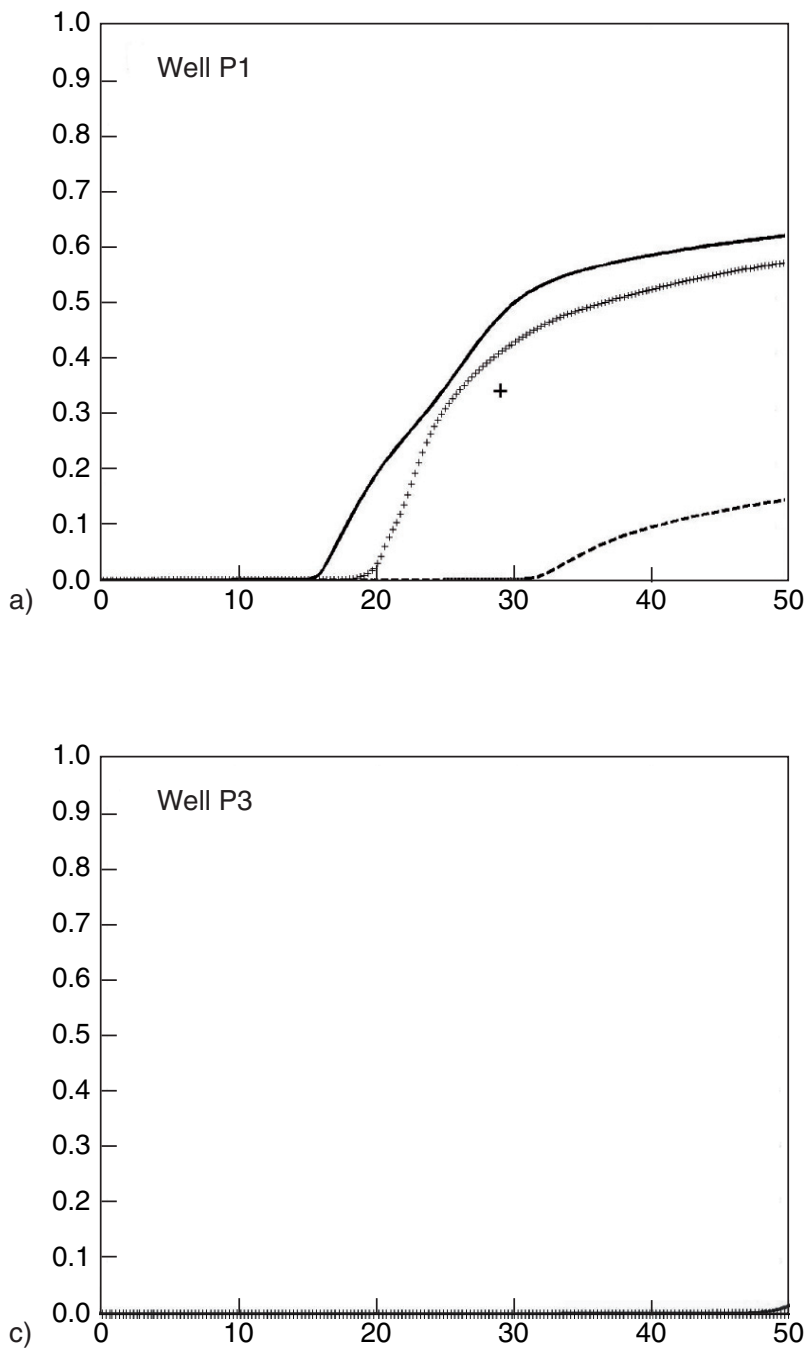

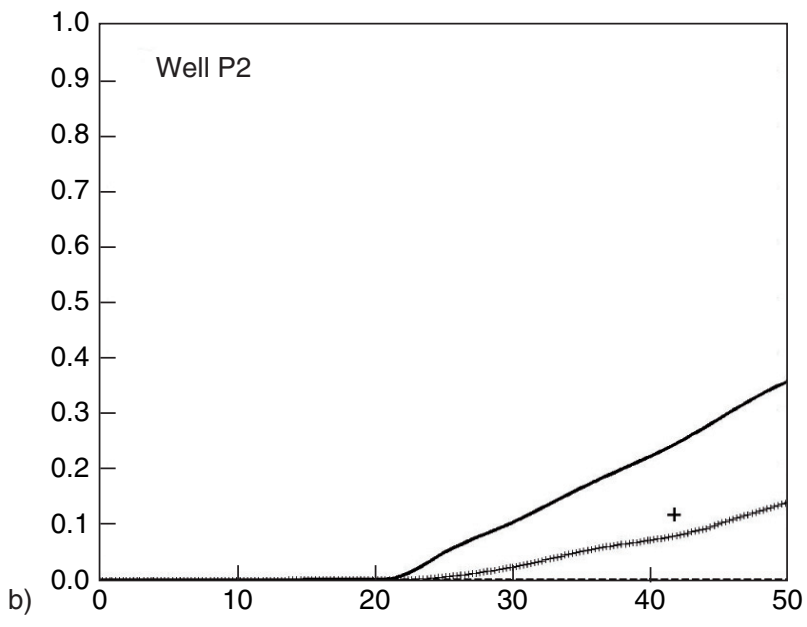

d)

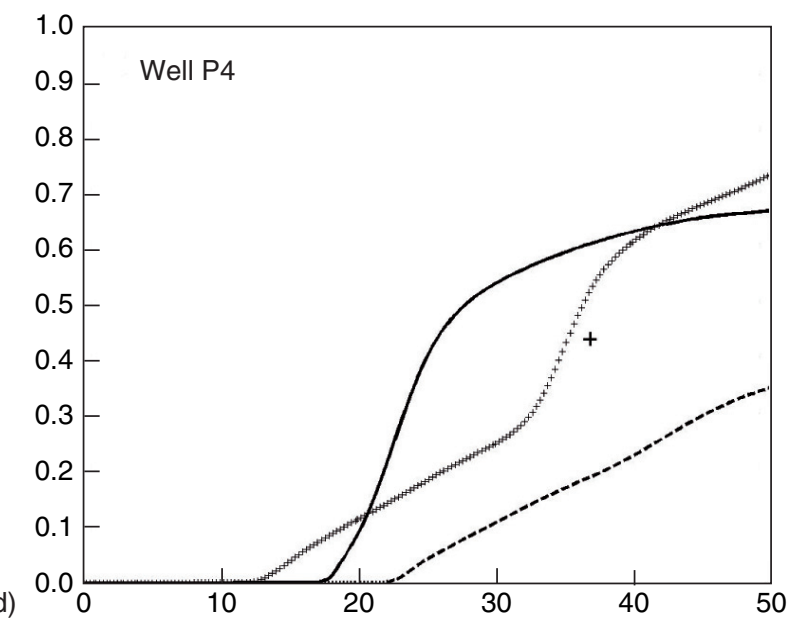

Figure 11

Calibration of the second realization: comparison of the water-cut curves of the reference network $(-)$, the initial realization $(--)$ and the realization after global optimization $(+++)$.

where:

$K_{\text {mat }}:$ matrix permeability,

$K_{\text {frac }}$ : fracture permeability,

$e_{\text {frac }}$ : fracture thickness,

$\phi_{\text {mat }}:$ matrix porosity,

$H$ : reservoir thickness.

These parameters are considered known and fixed in the following calibration procedure, although most of them, particularly the fracture permeability and thickness, are highly uncertain in practice. Our future work will also account for uncertainties of fracture petrophysical properties.

\subsection{Calibration of the Geometry of Fracture Network}

The stochastic model of the fracture network is composed of two independent fracture sets: one oriented N120-130 and the other N170. We build realizations of the stochastic model of fracture networks constrained to the fracture density map. Then, we perform their calibration to the reference water-cut data. The two fracture sets are calibrated simultaneously. There is one fitting parameter for each fracture set. The simplex method is used for this two parameter optimisation problem. In the following examples, the number of iterations of the simplex algorithm is limited to 20 for optimizing each realization chain. 


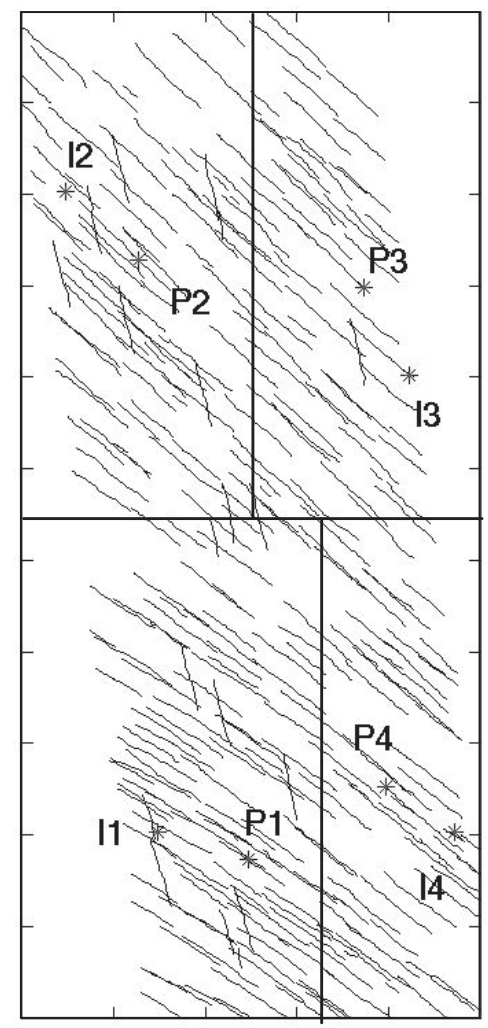

Figure 12

Calibration of the second realization: realization of the stochastic fracture network after global optimization.

The objective function is defined as the sum of the quadratic errors of the model response with respect to the reference response at the four wells:

$$
F=\frac{1}{2} \sum_{\text {wells }} \sum_{i=1}^{n}\left(d_{\text {model }}^{i}-d_{\text {ref }}^{i}\right)^{2}
$$

where:

$d_{\text {ref: }}^{i}:$ vector of reference water-cut data,

$d_{\text {ref }}^{i}:$ vector of water-cut data of the stochastic model.

Using the objective function (4), we give all data the same weight. But in general, different weights can be used for different data according to their importance with respect to the problem of interest.

The reference water-cut data were simulated on the reference network shown in Figure 4. These data stand for the actual field production history that would be used in practice.

\section{Calibration of a First Realization}

Let us consider the initial realization shown in Figure 1. The fluid flow simulation on this realization reveals a water-cut evolution very different from the reference case (Fig. 6). From this example, it is obvious that a realization constrained only to geological data does not necessarily reproduce the hydrodynamic behavior of the reservoir.

Then, we perform the global calibration of this initial realization, to water-cut data at the four wells. Figure 7 shows the evolution of the objective function when iteratively optimizing ten realization chains. We note that about $90 \%$ of the improvement was obtained by optimizing the first realization chain. From third realization chain, the calibration improvement becomes negligible. The fact that the first optimization is much more efficient than the following ones was also observed in different applications involving the gradual deformation algorithm [6].

Figure 6 compares, for each production well, the water-cut curves of the reference case, the initial and the optimal realizations. The calibration is satisfactory. Figure 8 shows the fracture network of the optimal realization.

\section{Calibration of a Second Realization}

Consider now another initial realization generated at random (Fig. 9), on which we apply the same procedure of history matching. Figure 10 shows the evolution of the objective function for five iterations. The calibration provides a general improvement of the model (Fig. 11), but not as good as in the first example. Figure 12 shows the fracture network of the optimal realization.

To further improve the match of each well, we can perform a local calibration. Accordingly, the reservoir field is subdivided into four zones and each zone contains an injector and a producer (Fig. 12). Alike the global calibration, there are two parameters (one for each fracture set) in each zone. The calibration is performed sequentially zone by zone. When a zone is calibrated, only the fracture locations (seeds) in this zone are retained. The propagation of fractures are performed for all seeds in the reservoir field each time when some fracture seeds are moved. This guaranties the spatial continuity of the fracture network model. Another possibility is to perform simultaneous calibration of the four zones. The objective function depends then 8 parameters ( 2 for each zone).

Figure 13 compares the water-cut curves of the reference case and of the optimal realization after local calibration. The production match is much better than through the previous global calibration of the four wells together. The fracture network of the locally calibrated realization is shown in Figure $14 \mathrm{c}$. This realization shares the general features (same orientation and density of fractures) with the initial realization (Fig. 14a) and with the realization after global calibration (Fig. 14b). But the location of each individual fracture is different from one realization to another. This makes the fracture network in Figure 14c history-matched, contrarily to the networks in Figures 14a and 14b. 
a)
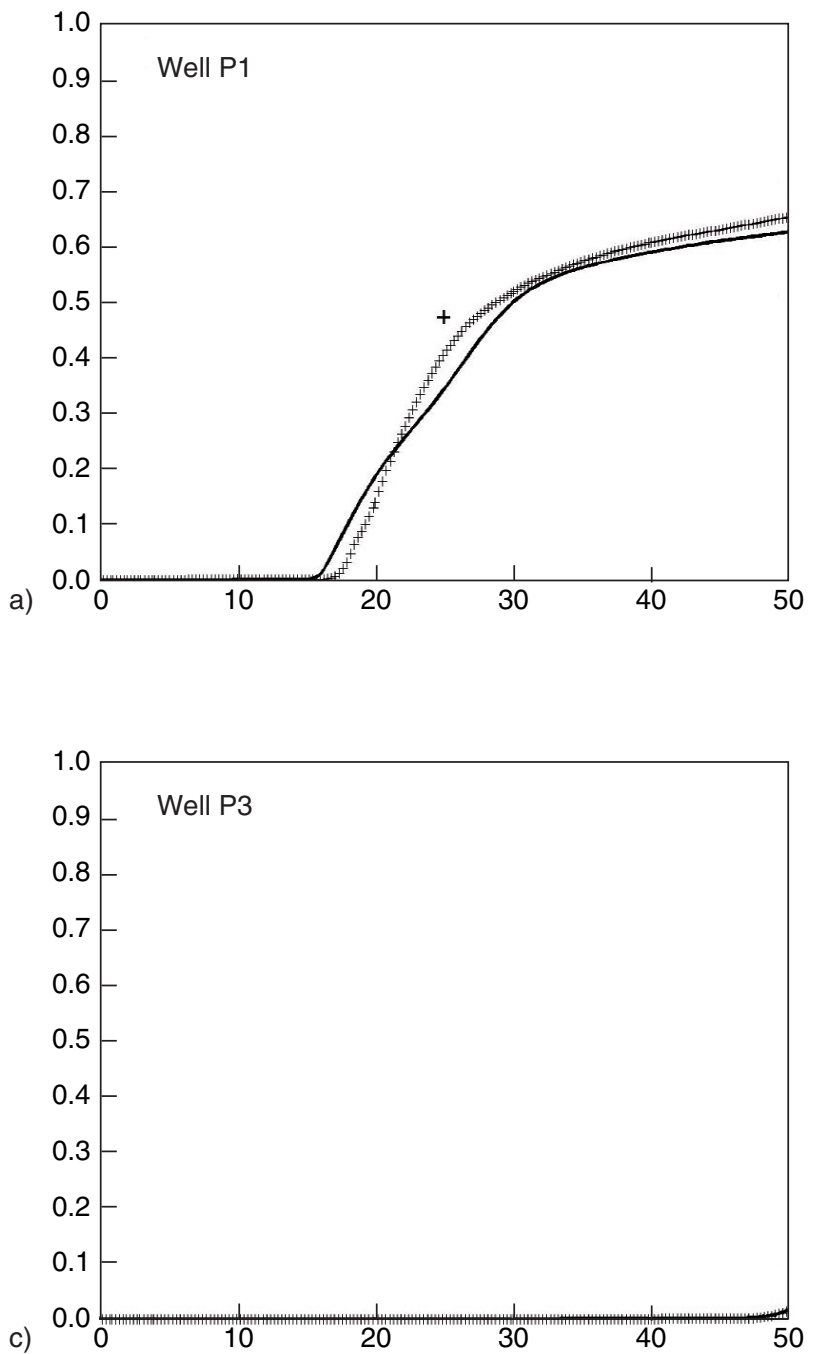

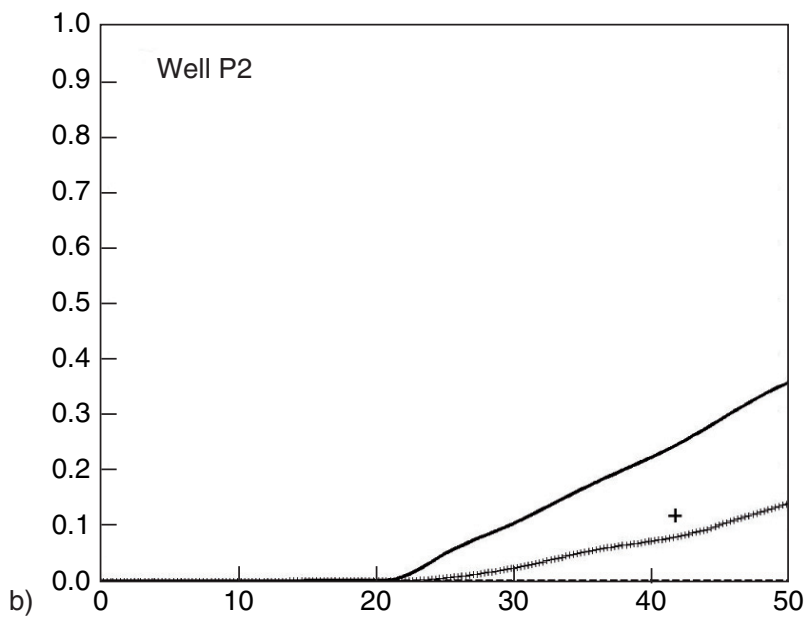

d)

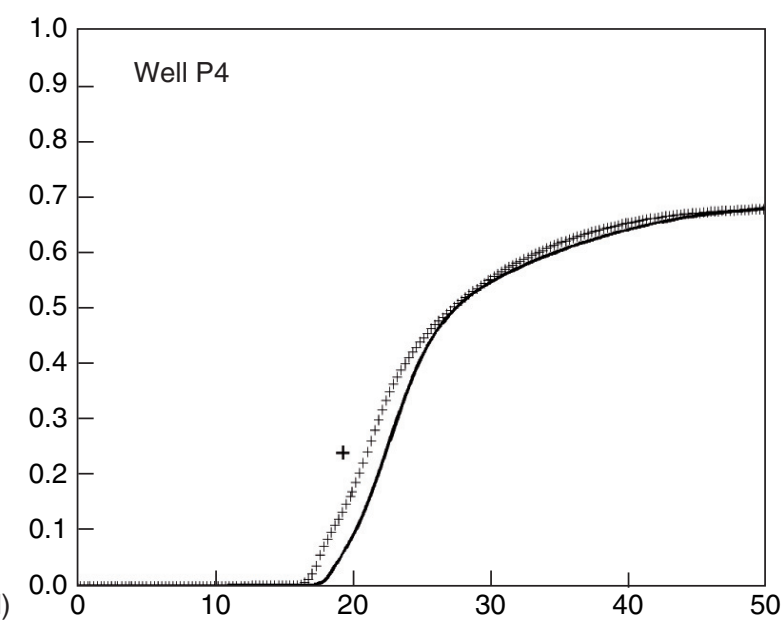

Figure 13

Calibration of the second realization: comparison of the water-cut curves of the reference network ( -$)$ and the realization after local calibration (+++).

\section{CONCLUSIONS}

In this work, we investigated the impact of geometrical properties of large-scale fractures on the dynamic behavior of fractured reservoirs. The main conclusions of this work are as follows:

- The object-based stochastic modeling procedure presented in this paper is able to describe the geological features of large-scale fractures, namely sub-seismic faults and fracture swarms.

- However, the local flow behavior simulated on the model realizations, constrained only to orientation and density maps of fracture sets, can still be far from that of the real reservoir. This justifies the necessity of a history matching step.

- The proposed history matching procedure, based on the gradual deformation method, preserves the model geological features, namely the orientation and density maps of fracture sets.

- The numerical results show that the global calibration procedure can be very efficient when optimizing the first few realization chains, but does not necessarily lead to a satisfactory history-matched realization. The local calibration procedure can further improve the history match in the different field sectors. 


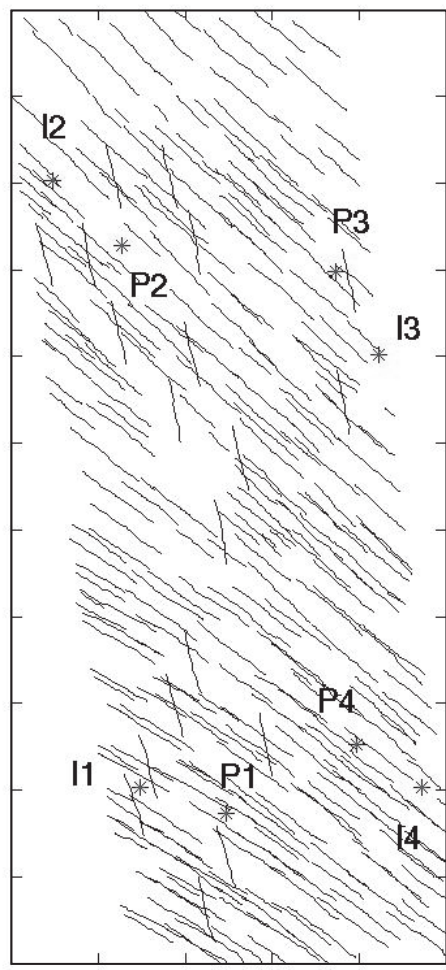

a) Initial realization

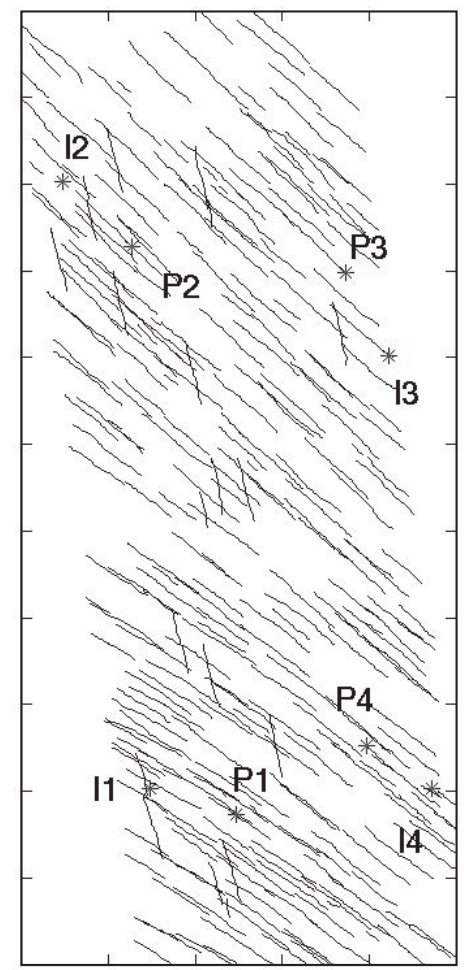

b) After global calibration

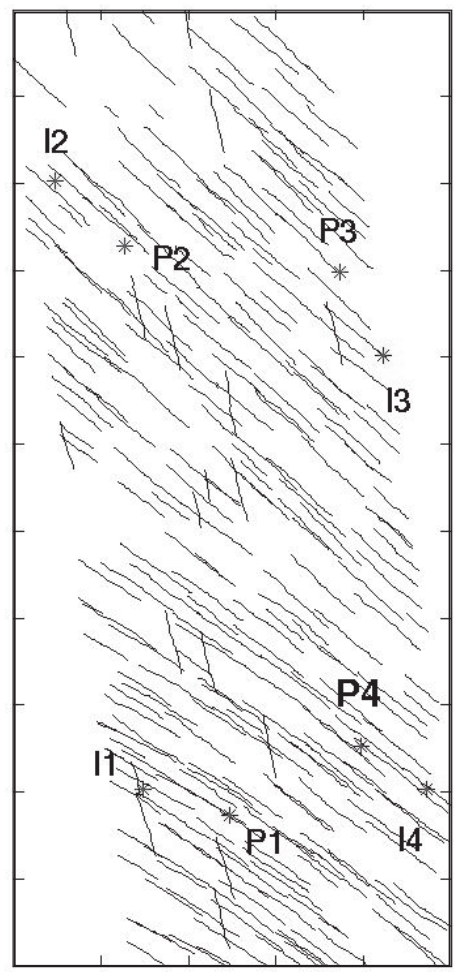

c) After local calibration

Figure 14

Calibration of the second realization: comparison between the initial realization, the realization after global calibration and the realization after local optimization.

- By repeating the history matching procedure from different initial realizations, one can obtain different calibrated realizations for uncertainty evaluation.

The present methodology is still limited to large-scale vertical fractures that cross the entire reservoir. Our ongoing research is on its extension to completely three dimentional fractures. Another important issue that is not considered here is the impact of petrophysical properties of large-scale fractures. This will also be addressed in our future work.

\section{ACKNOWLEDGEMENTS}

This work is partly supported by the companies: Repsol$Y P F$, Statoil and Total through the Calfrac Joint Industryfunded Project and partly by the European Community through the Marie Curie fellowship of Sandra Jenni under the contact HPMI-CT-1999-00012. We would like to thank Marie-Christine Cacas, Jean-Marc Daniel and Emmanuel Manceau (IFP) for helpful discussions. We are also grateful to the reviewers of this paper for their helpful comments.

\section{REFERENCES}

1 Chilès, J.P. and de Marsily, G. (1993) Stochastic models of fracture systems and their use in flow and transport modeling. In Flow and contaminant transport in fractured rock, J. Bear, G. de Marsily and C.F. Tsang (Eds.), Academic Press, San Diego, California, Chap. 4, 169-236.

2 Cacas, M.C., Daniel, J.M. and Letouzey, J. (2001) Nested Geological Modelling of Naturally Fractured Reservoirs. Petrol. Geosci., 7, S43-S52.

3 Bourbiaux, B., Cacas, M.C., Sarda, S. and Sabathier, J.C. (1998) A rapid and efficient methodology to convert fractured reservoir images into a dual porosity model. Oil Gas Sci. Technol., 53, 785-799.

4 Sarda, S., Jeannin, L., Basquet, R. and Bourbiaux, B. (2002) Hydraulic characterization of fractured reservoir: Simulation on discrete fracture models. SPE paper 66398. 
5 Cosentino, L., Coury, Y., Daniel, J.M., Manceau, E., Ravenne, C., Van Lingen, P., Cole, J. and Sengul, M. (2001) Integrated study of a fractured Middle East reservoir with stratiform super$\mathrm{K}$ intervals- Part 2: Upscaling and dual media simulation. SPE paper 68184.

$6 \mathrm{Hu}$, L.Y. and Jenni, S. (2005) History matching of object-based stochastic reservoir models. SPE J, 312-323.

7 Bourbiaux, B., Basquet, R., Cacas, M.C. and Daniel, J.M. (2002) An integrated workflow to account for multi-scale fractures in reservoir simulation models: Implementation and benefits. SPE paper 78489 .
8 Gauthier, B.D.M., Garcia, M. and Daniel, J.M. (2002) Integrated fractured reservoir characterization: A case study in a North Africa Field. SPE paper 79105.

9 Stoyan, D.S., Kendall, W.S. and Mecke, J. (1995) Stochastic geometry and its applications, 2nd Edition, Wiley, Chichester.

10 Press, W.H, Teukolsky, S. A., Vettering, W.T. and Flannery, B.P. (1992) Numerical recipes in C. Cambridge University Press.

Final manuscript received in October 2006 